\title{
Subsequent Entry Biologics in Canada: Current State of the Science
}

Laszlo Endrenyi ${ }^{1}$, Fakhreddin Jamali ${ }^{2}$, and Raimar Loebenberg 2

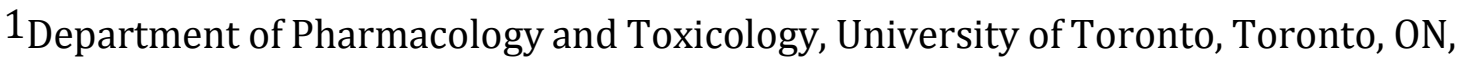
Canada; ${ }^{2}$ Faculty of Pharmacy and Pharmaceutical Sciences, University of Alberta, Edmonton, $\mathrm{AB}$, Canada

Corresponding author: Fakhreddin Jamali, Faculty of Pharmacy \& Pharmaceutical Sciences, University of Alberta, Edmonton, Alberta, Canada T6G 2E1 fjamali@ualberta.ca

\begin{abstract}
The Canadian Society for Pharmaceutical Sciences organized a workshop on the current state of sciences of subsequent entry biologics (SEBs, biosimilars) on December 10th 2014 in the Health Canada location in Ottawa, ON. The day-long workshop provided an opportunity to discuss recent regulatory developments and a wide range of scientific issues related to SEBs. Following a discussion on the differences between the Canadian guidance and those of other countries, a series of presentations were made that focused on the regulatory requirements with regard to the product quality, methodology, non-clinical and clinical data. In addition, issues of extrapolation from one indication to another, interchangeability and reimbursement were articulated. It was also highlighted that both the patients and caregivers need to be better informed regarding the safety and efficacy of articulated SEBs.
\end{abstract}

The Canadian Society for Pharmaceutical Sciences organized a workshop focusing on the current state of sciences of subsequent entry biologics (SEBs, biosimiars) on December 10 $0^{\text {th }}, 2014$ in the Health Canada location in Ottawa, ON. The day-long workshop provided an opportunity to discuss recent regulatory developments and a wide range of scientific issues related to SEBs. The workshop was attended by about 150 participants from various backgrounds including regulatory, academia, industry, legal and consumer advocacy.

\section{Overview of Workshop Presentations}

Agnes Klein, Director, Centre for the Evaluation of Radiopharmaceuticals and Biotherapeutic Products, Health Canada, opened the workshop and set the stage for the day by pointing out the complexity of SEB molecules compared to the chemistry of small molecule generics, and the need for scientific solutions for SEB regulation. While Health Canada has recently taken regulatory positions, which differ from other global regulators, it is generally agreed upon that the science must be at the center of such decisions and considerations. She posed four questions: 
1. Where are the gaps in sciences that would allow for a better regulation of SEBs?

2. What should be the level of comparability between the SEB and the reference biological product?

3. What should Health Canada consider in addition to the principles enunciated so far in the extrapolation of indications?

4. What are the gaps that we have in our SEB guidance that need to be considered when analyzing the guideline and that require updating?

Bobby Chauhan, Senior Regulatory Officer, Biologics and Genetic Therapies Directorate, Health Canada, provided an overview of Biologics and SEBs, and the relevance regulations under the Food and Drug Regulations in Canada.

SEBs must undergo a full drug submission process, which is different from the Abbreviated New Drug Submission pathway for generic drugs. SEBs cannot claim "pharmaceutical equivalence", "therapeutic/clinical equivalence", or "bioequivalence". SEBs are regulated like all "new drugs" under the Food and Drug Regulations.

Health Canada's 2010 Guidance for Sponsors: Information and Submission Requirements for Subsequent Entry Biologics (SEBs) addresses three main pillars: (i) quality (including similarity between the SEB and reference biologic drug), (ii) non-clinical, and (iii) clinical studies. It is up to the sponsor to provide the necessary evidence to support all aspects of an application for authorization which applies especially to the similarity of products but also the risk of immunogenicity.

Health Canada is in regular contact with other global health authorities to exchange viewpoints on biologic drugs. These include the World Health Organization, the Asia-Pacific Economic Cooperation, and the International Pharmaceutical Regulators Forum. In addition, the European Medicines Agency, the US Food and Drug Administration and Health Canada hold quarterly meetings to discuss issues related to SEBs.

As the scientific landscape evolves for SEBs, Health Canada looks to revise the SEB Guidance. Some areas identified for review or discussion include: extrapolation to other indications; spectrum of similarity; defining the most sensitive population and clinical endpoints; use of more than one non-Canadian reference product in clinical studies; link between the SEB, non-Canadian reference and Canadian reference biologic drug; immunogenicity assessments; interchangeability/ substitutability and international Non Proprietary Names (INN) standards.

Jeffrey Skene, Chief, Monoclonal Antibodies Division, Health Canada, presented on Quality Issues and Comparability in Subsequent Entry Biologics. SEBs can get market authorization within the scope of current regulations. An "Outline Document" on the Canadian regulatory approach to SEBs has been available since 1999 and a "Fact Sheet" on SEBs has been available since 2006. Health Canada held external consultations and a workshop in 2008. The final guidance was released in 2010. Health Canada uses a comparability approach for quality, non-clinical and clinical studies to support the similarity of products. In Europe, a biosimilar must demonstrate similarity to the reference medicinal product in terms of quality characteristics, biological activity, safety and efficacy based on a comprehensive comparability exercise. The US FDA requires manufacturers to perform in-depth chemical, physical, and bioactivity 
comparisons with side-by-side analyses of an appropriate number of lots of the proposed biosimilar product and the reference product. In Canada the SEB must be "subsequent" to a product approved for the Canadian market but non Canadian reference products may be used; the product must meet the criteria for similarity / comparability. This can be provided by "quality" studies, which may include suitable data on safety and effectiveness for the reference biologic product. Analytical techniques should characterize the product using more than one method to characterize a product attribute. Appropriate acceptance criteria must be provided and stability data should support similar degradation processes. A side-by-side stress-test is recommended; differences must be justified. Health Canada has adopted a regulatory approach to SEBs that is science-based; however, the International Conference of Harmonization guidance documents should be used where possible. The regulator is actively pursuing opportunities for collaborative regulatory approaches and relevant guidance from other regulatory partners will be referenced or adopted as appropriate.

Bob Burgoyne, Director, Life Sciences Business Development, Waters Corp., focused his presentation on the analytical techniques which are available today. Bioanalysis means physicochemical characterization, but not bioassays. Comparability studies can utilize modern analytical methods, but only clinical studies will provide evidence of similarity in regard to clinical outcomes.

Critical quality attributes can be primary structure, post-translational modifications, chemical modifications (e.g. oxidation, deamidation), disulfide linkage pattern, glycosylation pattern, aggregation, charge variant pattern, host cell protein impurity and protein conformation. All of this can influence efficacy, immunogenicity, ADME properties, pharmacokinetics and product (shelf life) stability. He gave the example of infliximab for a comparability study for a biosimilar against the innovator.

The molecule of interest was analyzed by different analytical techniques and the entire molecule as well as molecular fragments e.g. light and heavy chains were investigated for their similarity. Intact protein mass Spectroscopy can reveal major glycosylation forms and lysine variants of an antibody. This is important because glycans impact safety and efficacy of a biologics. A correct and consistent structure of the glycans is important to obtain the desired medical effect and to avoid adverse immunological reactions. Light or heavy chain analysis gives an in-depth knowledge of the similarity between the innovator and SEB for each molecular fragment.

Modern analytical techniques can provide a new totality of evidence, which was not available some year ago.

Gopi Shankar, Head, Bioanalytical Sciences \& Immunogenicity, Janssen Research and Development, LLC, presented on immunogenicity assays. Dr. Shankar showed that biochemical/biophysical characterizations and predictive immunogenicity analysis have been improved over the years but cannot yet, and perhaps never, substitute for clinical immunogenicity testing. Clinically relevant is that a SEB has similar immunogenicity compared to the reference product. It is required to demonstrate that the immunogenicity of the proposed product is not increased in comparison with the reference product (i.e., a non-inferior safety profile relative to a reference immunogenicity). Immunogenicity to an antigen is epitope specific and an anti drug 
antibody assays can be used for testing for this. Anti-drug antibody methods should not involve the use of calibrator standards because there is no "standard". Instead a "cut-point" and titers should be used. In humans, anti-drug antibodies could be expressed in IgG, IgM, IgA and IgE, with kappa or lambda light chains, and so ADA assays should be able to detect all isotypes. The assay development depends upon the availability of an analyte (a positive control). Assay specificity and sensitivity, in the presence of drug and target, are key factors that influence the accuracy of immunogenicity results.

Methods have to be cautiously developed to avoid false positive and false negative results. If a single SEB-based method is used (for ADA capture and detection), when applied to the reference arm of a study, detects cross-reactivity only. This approach assumes that the SEB is antigenically identical to the reference, or has a lower immunogenicity.

The comparison is only valid if a SEB based method and a reference based method are both applied, to the appropriate arms of the study, or ideally to both arms.

Jian Wang, Chief, Clinical Evaluation Division, Biologics and Genetic Therapies Directorate, Health Canada, presented on the topic of clinical development.

The goal of the clinical program is to resolve residual uncertainties regarding whether limitations and differences in the quality data between a SEB and a reference product could result in clinically meaningful differences in regard to product efficacy or safety and immunogenicity. Comparative PK/PD studies can be used to demonstrate comparability to justify a reduction of subsequent clinical studies. A 95\% confidence interval should be used for comparative PK/PD studies, but for many biologics, especially mAbs, no relevant PD surrogate might exist. Equivalence trials are preferred over non-inferiority trials and based on the ICH definition, an equivalence trial is a trial designed to show that two interventions do not differ in either direction by more than a pre-specified insignificant margin that can be judged as clinically acceptable.

A risk based immunogenicity evaluation strategy should be used where the SEB is compared against the reference product using a sufficient number of patients and sufficient study duration. A post-marketing risk management plan is required for SEBs.

Unlike generic drug products, SEB sponsors are not able to use the entire product monograph of the reference products. Also there should be no claims for bioequivalence between the SEB and reference product. The product label is a combination of information of relevant safety data from the reference product and the SEB. Extrapolation depends on comprehensive comparative product characterization and demonstrated product similarity. Extrapolation between two indications with very different immunogenic profiles is not considered. In most cases monotherapy is recommended to support extrapolation, however, extrapolation of indications is not considered if the comparability between the reference and the SEB is insufficient. The SEB infliximab for example is comparable to Remicade in situations where the mechanism of action depends exclusively on its binding to TNF $\alpha$. Where the mechanism of action is not clearly defined or cannot be conclusively determined extrapolation is not allowed. Decisions are made based on the totality of evidence that is submitted. A Supplementary New Drug Submission (SNDS) can be filed for a SEB to 
support label changes and extrapolation of clinical data provided the information package filed is scientifically supportive and any provided rationale is sufficiently persuasive.

Michael Guirguis, the Drug Stewardship Pharmacist, Alberta Health Services, shared the pharmacist and patient perspective of how medication decisions might be made for SEBs.

Interchangeability decisions or substitutions are handled at the national level in Europe. SEBs are considered to be therapeutic alternatives to their reference products. The FDA considers SEBs interchangeable biological products if the risk in terms of safety or diminished efficacy of alternating or switching between the SEB and the reference product is not greater than the risk of using the reference product without such alternation or switching. Designation of interchangeability is a provincial matter in Canada. SEBs are not considered to be therapeutically or pharmaceutically equivalent to the reference biologic drug. Health Canada does not support the automatic substitution of an SEB for its reference product. Therefore, patients in different countries depend on the local definition of "interchangeable" and a pharmacist without the intervention of a health care provider might be required to substitute biologics. Thus interchangeability might cause automatic substitution. According to the European Generic Medicines Association, more than 12 countries across Europe have introduced rules to prevent automatic substitution of biological medicines.

According to Health Canada, the substitution of an SEB for a reference product can only be seen as a therapeutic substitution. However, in hospital settings therapeutics substitution is a common practice. Clarity has yet to be achieved regarding the SEBs in pharmaceutical care. What needs to be resolve is the required set of steps for pharmacist to switch a patient from a reference to a SEBs. Another question is whether payers can require a switch to occur.

Anthony S. Russell, of the Division of Rheumatology at the University of Alberta, gave clinical insights about extrapolation of indications. He started with the statement that for many biologics the sensitivity/reliability of the outcome measures are relatively poor compared to peptides like insulin and its effect on blood glucose. For example, some anti TNF agents bind monoclonal antibodies and neutralize soluble TNF-a and TNF-b, but not membrane-bound TNF-a. Therefore, the same drug may work on only some diseases indicating that a common single pathophysiology does not always seem to exist and, hence, this makes the validity of indication extrapolation questionable. Side effects of the same drug may differ in different populations and the same drug works for certain diseases but not for others. For example, infliximab helps in rheumatoid disease such as arthritis, but it does not work for pulmonary fibrosis. However, both conditions are linked to TNFs. The pathophysiology, therefore, has to be well understood before any extrapolation can be done.

He supports the decisions of Health Canada to approve only certain indications.

Another example is potential impact of manufacturing differences. Glycosylation might be different with different processes resulting in similar products, but not identical ones. This has implications for safety, immunogenicity and potency. 
Considerable uncertainty exists about the extent to which the Fc-domain glycosylation of infliximab impacts the mechanism(s) of action in different indications- and different aspects of the same indication.

SEBs, therefore, are not interchangeable with each other or with brand name biologics. Each biologic product must have a unique product name given the fact that SEB products are not identical to innovator products and could have significantly different clinical outcomes.

Strict post-marketing surveillance must be followed.

Michael S. Reilly, Executive Director, the Alliance for Safe Biologic Medicines, presented the data of the Canadian Physician Survey, a project jointly commissioned by the ASBM and BIOTECanada. The project surveyed 427 physicians from four provinces in Canada (Alberta, British Columbia, Ontario and Quebec). The survey's objectives were to assess the implications of a SEB sharing a nonproprietary name with its reference innovator product, to determine (i) how physicians identify biologics in patient records and in adverse event reporting, (ii) to gather physician perspective on the importance of distinguishable naming and (iii) to gauge physician attitudes on pharmacy substitution. The survey participants covered a wide field of expertise including allergy, immunology, infectious diseases, endocrinology, nephrology, urology, neurology, rheumatology, respiratory/ pulmonology, oncology, gastroenterology, internal medicine and dermatology. 35\% came from community settings and 33\% from academic centers the rest practices in multi-specialty clinic, private or family practice.

$48 \%$ were familiar SEB or had a basic understanding but only $10 \%$ had in-depth knowledge of them. 52\% were aware that a SEB may be approved for several or all indications of the reference product on the basis of clinical trials in only one of those indications. $62 \%$ thought that if two medicines have the same non-proprietary scientific name, either product could be given with the same expected results. $76 \%$ believed that if two biologic medicines have the same non-proprietary name, it suggests that the medicines are approved for the same indications. 54\% thought a completely different INN for SEBs was the best way for Health Canada to differentiate a SEB from the innovator biologic. 71\% thought it was not acceptable for the pharmacist to make the determination which biologic to dispense.

The survey identifies a need for additional education and information on SEBs among Canadian physicians. It also highlighted the potential confusion that can arise if both the SEB and the innovator product have the same INN. Misconceptions about SEBs, along with physician prescribing and recording practices, highlight the need for a distinguishable naming scheme for all biologics, including SEBs.

David Zante, Director, Health Economics \& Reimbursement, Janssen Inc., presented on reimbursement considerations.

Most provinces participate in the Common Drug Review (CDR). Stakeholders and patient groups can give input to this formalized process. The goals of the CDR are harmonized listing criteria and getting better drug prices. SEBs undergo this process. The CDR SEB submission template has differences compared to innovative Drug Submissions. For example, a reduced clinical data package is acceptable for SEBs, but 
the "Focus on the key results that were used to justify the reduced non-clinical and clinical data packages for the SEB" is needed. The SEB submission template contains cost tables - essentially a cost comparison between SEB and reference product (under the assumption of equivalent efficacy/safety). The extrapolation section allows inclusion of positions from foreign regulatory agencies in the template. Patient expectations on the use of the SEB can be included, such as possible switching approaches and what this might mean for a patient. However, CDR does not deal with interchangeability or substitution - this is determined at the provincial level. In Quebec, INESSS, not CDR, makes a recommendation to the Minister. The new Pan Canadian Pharmaceutical Alliance (pCPA) will use the CDR recommendation. pCPA negotiations may create one of two different scenarios: (i) With a modest discount compared to the reference drug's Product Listing Agreement price, resulting in market competition, or (ii) with significant discounts compared to the reference drug's Product Listing Agreement price. Scenario 2 may result in a "Use SEB first" listing on formularies, for new patients, but existing patients are expected to be maintained on the brand product. Private insurers are also following these issues closely with the appreciation that SEBs are not "generic biologics".

The workshop ended with a discussion between the audience and a panel consisting of the speakers and Durhane Wong-Rieger of the Canadian Organization for Rare Disorders who highlighted the gap that exists between the scientists and the consumer.

\section{Organizing Committee:}

Bobby Chauhan (Health Canada), Laszlo Endrenyi (University of Toronto), Fakhreddin Jamali (University of Alberta), Agnes Klein (Health Canada), Cathy Lau (Janssen), Raimar Loebenberg (University of Alberta), Iman Mohamed (BIOTECanada), Don Wallar (Apotex), Barbara Scollick (CSPS)

\section{Sponsors:}

Janssen Inc. - 19 Green Belt Drive, Toronto, ON

Apotex Inc. - 150 Signet Drive, Toronto, ON

BIOTECanada - 600-1 rue Nicholas Street, Ottawa, ON 